Cognitive Functional Therapy: An Integrated Behavioral Approach for the Targeted Management of Disabling Low Back Pain

\title{
Kognitive funktionelle Therapie: Ein integrierter Verhaltensansatz zur gezielten Behandlung von körperlich behindernden Schmerzen des unteren Rückens
}

O'Sullivan PB, Caneiro JP, O'Keeffe M, Smith A, Dankaerts W, Fersum K, O'Sullivan K. Physical Therapy 2018; 98 (5): 408-423. DOI: 10.1093/ptj/pzy022

\section{S. Bechter}

\section{Zusammenfassung}

\section{Ziel}

Dieser Artikel beschreibt, wie die Methode der kognitiven funktionellen Therapie (KFT) bei Patienten mit körperlich behindernden, unspezifischen chronischen lumbalen Rückenschmerzen (NSCLBP) angewendet werden kann. Nebst den Komponenten, die diese Therapiemethode charakterisieren, wird deren Wirkung anhand von drei Fallbeispielen illustriert.

\section{Hintergrund}

NSCLBP, weltweit die Hauptursache für körperliche Behinderung, werden, wenn sie nicht auf eine entsprechende Pathologie zurückzuführen sind, durch veränderte neurobiologische Prozesse und Verhaltensanpassungen des Patienten (unter anderem Vermeidung körperlicher Aktivität aus Angst vor Schmerzausweitung) erklärt. Sie werden durch individuelle Gesundheitsfaktoren (physische, psychologische, soziale, (epi-)genetische, LifestyleFaktoren, etc.) beeinflusst. Die Prävalenz von NSCLBP ist zunehmend, steigende Gesundheitskosten sind die Folge.

Biomedizinische Ansätze reichen zur Diagnose und erfolgreichen Behandlung von NSCLBP nicht aus. Verschiedene Ansätze, wie zum Beispiel das Klassifizierungs- und Subgruppierungssystem scheinen zu eindimensional und reduktionistisch zu sein und die biopsychosozialen Aspekte zu wenig zu berücksichtigen ${ }^{1}$. Aus diesem Grund wurde die KFT eine flexible, integrierende Methode zur Diagnose und individualisierten Behandlung von NSCLBP entwickelt. Sie soll es ermöglichen, den Patienten aus dem Teufelskreis «Schmerz», an dem verschiedene Faktoren beteiligt sind, herauszuführen. Das Verhalten und die Verhaltensänderung stehen dabei im Zentrum. Die KFT soll Patienten dabei unterstützen, den Schmerz in ihrem persönlichen Kontext zu verstehen und unter Berücksichtigung ihrer individuellen Ziele einen Schmerzmanagementplan zu entwickeln.

\section{Methode}

Die Autoren erläutern in ihrem Artikel die Bedeutung multidimensionaler Faktoren, die mit NSCLBP assoziiert werden. Sie zeigen auf, was es bei einem umfassenden Assessment zu beachten gilt. Das Erfassen der Schmerzcharakteristika kann helfen, die individuelle Präsentation von Schmerzen zu verstehen und die Behandlung entsprechend zu gestalten. Mit pathoanatomischen Befunden aus bildgebenden Verfahren, wie sie auch bei vielen beschwerdefreien Personen vorkommen, soll sorgfältig umgegangen werden, um den Patienten nicht zu verunsichern. Auf ein verändertes Bewegungsverhalten (übervorsichtige 
Bewegung, Angst vor Bewegung, Bewegung begleitet von sympathischen Reaktionen wie Schwitzen, oberflächlicher Atmung etc.) soll geachtet werden. Die psychologischen Faktoren - kognitive (Einstellung zum Schmerz) und emotionale (Distress, Angst) - müssen besonders berücksichtigt werden, da sie eine Schmerzausweitung und -persistenz begünstigen können. Der kulturelle und soziale Hintergrund des Patienten sowie seine Lebensgeschichte (schwierige, prägende Ereignisse) müssen, auch wenn sie nicht verändert werden können, in die Behandlung einbezogen werden. Weiter sollen Lifestylefaktoren wie Schlaf, Aktivitätslevel, abdominelles Übergewicht und Rauchen sowie generelle Gesundheitsfaktoren (Komorbiditäten) berücksichtigt werden.

\section{Ergebnisse}

Basierend auf den oben beschriebenen multidimensionalen Faktoren entwickelten die Autoren die KFT, welche sich als individualisiertes Management von Patienten mit NSCLBP versteht. Im Zentrum der Behandlung steht das Selbstmanagement des Patienten mit einem auf dessen klinische Präsentation und Kontext zugeschnittenen Programm. Die sogenannte therapeutische Allianz, charakterisiert durch eine offene, empathische und motivierende Haltung, sowie eine reflektierende und bestätigende Kommunikation, sind dabei von zentraler Bedeutung.

Die individuellen Faktoren wie Schmerzgeschichte, -verhalten, und -erleben, allfällige Befunde aus bildgebenden Verfahren, Kontextfaktoren, Erwartungen und Präferenzen sowie Selbstwirksamkeit des Patienten werden in der Anamnese erörtert. Im funktionellen Verhaltensassessment werden die in der Anamnese genannten schmerzhaften Haltungen, Bewegungen und Aktivitäten, welche unter Umständen vermieden werden oder mit Angst verbunden sind, untersucht. Eine sorgfältige Anleitung und Beobachtung der Bewegungsausführung und allfälliger sympathischer Reaktionen sind dabei besonders wichtig. Durch geschickte Führung kann der Patient erste positive Bewegungserfahrungen machen. Jahrelang vermiedene Bewegungen können so - unter fachkundiger Anleitung plötzlich wieder ermöglicht werden. Die eigentliche Behandlung besteht aus den folgenden drei Komponenten:

1. «Making sense of pain»: Anhand der eigenen Geschichte und der im Verhaltensassessment durchgeführten Bewegungen wird mit dem Patienten über den Teufelskreis «Schmerz», Bewegungsvermeidungsverhalten, Distress und körperliche Behinderung reflektiert. Dabei werden Kontextfaktoren, negative Überzeugungen sowie nicht hilfreiche emotionale und Verhaltensreaktionen erörtert. Weiter wird elaboriert, wie dieser Teufelskreis durchbrochen werden kann, um zu einem körperlich aktiveren Leben zurückzufinden.

2. «Exposure with control»: Damit ist der Prozess der Verhaltensänderung gemeint. Patienten werden behutsam an ursprünglich schmerzhafte, von Angst begleitete oder vermiedene Bewegungen herangeführt. Sie können positive Bewegungserfahrungen machen, ihre Selbstwirksamkeit steigern und sich so schrittweise im geschützten Rahmen ihren gesetzten Zielen (von ihnen festgelegte Aktivitäten) ohne Schmerzeskalation nähern.

3. «Lifestyle change»: Unter Berücksichtigung der Präferenzen und Möglichkeiten (u.a. Kosten, Erreichbarkeit von Angeboten) wird mit dem Patienten ein Aktivitätsprogramm zusammengestellt, welches ihn zu einer nachhaltige Verhaltensänderung motiviert.

\section{Schlussfolgerungen}

KFT, eine auf einem multidimensionalen Verständnis von Schmerz basierende Methode, kann zur Behandlung von Patienten mit NSCLBP eingesetzt werden. Bei Bedarf soll sie, so die Autoren, in ein interdisziplinäres Behandlungssetting integriert werden. 


\section{Kommentar}

Dieser Artikel erläutert auf verständliche Art und Weise, wie mit der KFT auf die Komplexität der Beschwerden und die Individualität von Patienten mit NSCLBP eingegangen werden kann. Die beschriebenen drei Fallbeispiele illustrieren eindrücklich, wie der Teufelskreis «Schmerz» mittels einer gezielten, individualisierten Behandlung mit einer geringen Anzahl Sitzungen (drei, fünf bzw. acht über drei Monate verteilt) durchbrochen werden kann. Es kann angenommen werden, dass die KFT damit einen Beitrag zur Senkung der Prävalenzrate von NSCLP und zur Kostensenkung im Gesundheitswesen leisten kann.

Es stellt sich allerdings die Frage, über welche Voraussetzungen die Patienten verfügen müssen, um von einer Behandlung mittels KFT langfristig profitieren zu können. Es ist anzunehmen, dass Faktoren wie Selbstwirksamkeit, Motivation und Unterstützung durch Angehörige - um nur die allgemein bekannten zu nennen - einen entscheidenden Einfluss auf den langfristigen Therapieerfolg haben.

Weiter wirft es die Frage auf, über welche Kompetenzen Physiotherapeuten verfügen müssen, um diese Methode erfolgreich anzuwenden. Nebst einer fundierten Befundaufnahme zur Identifizierung von unspezifischen Rückenschmerzen und zum Ausschluss ernsthafter Pathologien, bedarf es ausgeprägter Kommunikationsskills, die in das Fachgebiet der Psychologie hineinragen und im Curriculum der Grundausbildung der Physiotherapie nur marginal abgedeckt werden können. Nebst Berufserfahrung scheint eine fundierte Weiterbildung in der Methode der KFT, unabdingbar. In Australien und Norwegen, wo an der Wirksamkeit von KFT intensiv geforscht wird ${ }^{2}$, wird diese im Rahmen einer rund 100-stündigen Weiterbildung angeboten. Ein regelmässiger Austausch unter Kollegen in Form von Intervision oder Supervision könnte ein weiterer Schlüssel zum Erfolg dieser Methode sein.

Aufgabe der Bildungsverantwortlichen wird es sein, diese Methode in den Curricula zu verankern, und so zum Transfer von Erkenntnissen aus der Forschung in die Praxis beizutragen. Eine neue Methode implementiert sich bekanntlich nicht von selbst.

\section{Susann Bechter, MScPT}

Dept. Gesundheit, Institut für Physiotherapie ZHAW - Zürcher Hochschule für Angewandte Wissenschaften,Technikumstr. 71, 8401 Winterthur, Schweiz

susann.bechter@zhaw.ch

\section{Referenzen}

1 Karayannis NV, Jull GA, und Hodges PW. Physiotherapy movement based classification approaches to low back pain: comparison of subgroups through review and developer/expert survey. BMC Musculosceletal Disord. 2012; 13:1-24

2 Vibe Fersum K, O'Sullivan P, Skouen JS, Smith A, Kvåle A. Efficacy of classification-based cognitive functional therapy in patients with non-specific chronic low back pain: A randomized controlled trial. Eur J Pain. 2013; 17:916-928 